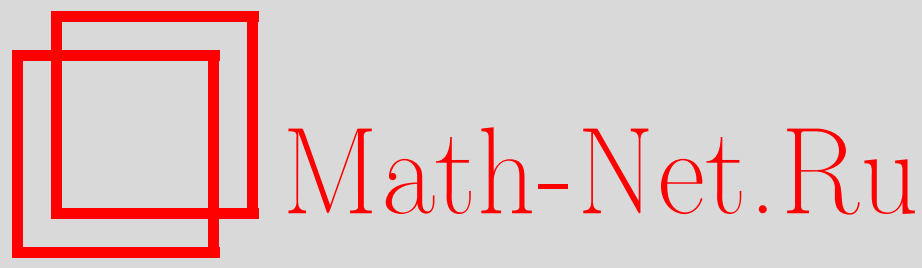

В. И. Кляцкин, Кластеризация положительного случайного поля как закон природы, ТМФ, 2013, том 176, номep 3, 494-512

DOI: https://doi.org/10.4213/tmf8538

Использование Общероссийского математического портала Math-Net.Ru подразумевает, что вы прочитали и согласны с пользовательским соглашением http://www.mathnet.ru/rus/agreement

Параметры загрузки:

IP : 54.224 .187 .69

26 апреля 2023 г., 12:09:27

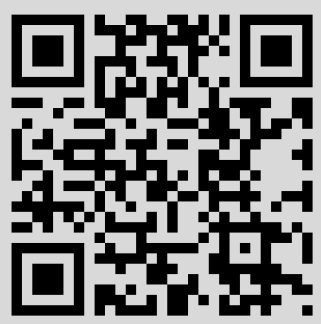




\title{
ФИЗИКА
}

Том 176, № 3

сентябрь, 2013

(C) 2013 г.

\author{
В. И. Кляцкин
}

\section{КЛАСТЕРИЗАЦИЯ ПОЛОЖИТЕЛЬНОГО СЛУЧАЙНОГО ПОЛЯ КАК ЗАКОН ПРИРОДЫ}

В параметрически возбуждаемых стохастических динамических системах могут с вероятностью единица образовываться пространственные структуры (кластеризация) почти в каждой реализации благодаря редким событиям, происходящим с вероятностью, стремящейся к нулю. Такие задачи возникают в гидродинамике, магнитной гидродинамике, физике плазмы, астрофизике и радиофизике.

Ключевые слова: перемежаемость, ляпуновский характеристический параметр, динамическая локализация, статистическая топография, кластеризация.

DOI: $1010.4231 / \operatorname{tmf} 8538$

\section{1. ВВЕДЕНИЕ}

Прежде всего сформулируем основную задачу статистического анализа стохастических динамических систем так, как мы ее понимаем: на основе статистического анализа вылвить общие особенности этих систем, которые осуществляются с вероятностью единица, т.е. почти для всех реализаций рассматриваемых динамических систем.

Такие процессы и явления, происходящие с вероятностью единица, будем называть когерентными. Подобную "статистическую когерентность" можно рассматривать как некую организацию сложной динамической системы, и выделение ее статистически устойчивых характеристик аналогично понятию когерентности как самоорганизации многокомпонентных систем, возникающей из хаотических взаимодействий их элементов (см., например, монографию [1]).

Заметим, что даже в гауссовых случайных полях могут осуществляться нетривиальные ситуации! Такие явления имеют место, например, в двумерных задачах геофизической гидродинамики вращающейся жидкости со случайной топографией дна (см. работы [2]-[4]). Так, для однослойной двумерной гидродинамической среды (баротропный поток жидкости) могут образовываться стохастические структуры с одним фиксированным масштабом длины, в случае двуслойного потока жидкости

${ }^{*}$ Институт физики атмосферы им. А. М. Обухова РАН, Москва, Россия. E-mail: klyatskin@yandex.ru 
(бароклинный поток) могут образовываться структуры с двумя постоянными масштабами длины. Примеры таких структур, по-видимому, были обнаружены как на экспериментальных установках на вращающихся платформах, так и при наблюдениях из космоса за состоянием морского волнения (см., например, работы [5]-[12]).

В настоящей работе мы ограничиваемся анализом стохастических динамических систем со случайным параметрическим возбуждением. Такие системы возникают во многих областях физики и могут описываться как обыкновенными дифференциальными уравнениями, так и уравнениями в частных производных. При этом параметрическое возбуждение, с одной стороны, сопровождается возрастанием со временем всех традиционных статистических характеристик решения задачи типа моментных и корреляционных функций любого порядка. С другой стороны, наряду с этим в отдельных реализациях случайных процессов и полей могут происходить такие стохастические нестационарные явления, как перемешивание, локализация и кластеризация в фазовом и физическом пространствах.

Кластеризация какого-либо поля - это возникновение компактных областей с большими величинами данного поля на фоне окружающих областей с относительно низкими их значениями. При статистическом усреднении вся информация о кластеризации, естественно, пропадает.

Прежде всего возникает вопрос: осуществляются ли такие явления как локализация и кластеризация, в отдельных реализациях рассматриваемых процессов и полей или нет, а если осуществляются, то за какое характерное время (или на каких характерных масштабах)?

Само явление структурообразования в стохастических параметрически возбуждаемых динамических системах хорошо известно в физике. Так, например, решения одномерных задач с параметрическим возбуждением, которые описываются обыкновенными дифференциальными уравнениями, являются случайными процессами. Простейшая такая динамическая система определяет логнормальный случайный процесс $y(t ; \alpha)$, задающийся обыкновенным стохастическим дифференциальным уравнением первого порядка

$$
\frac{d}{d t} y(t ; \alpha)=\{-\alpha+z(t)\} y(t ; \alpha), \quad y(0 ; \alpha)=1,
$$

где $z(t)$ - гауссов случайный процесс типа белого шума с параметрами

$$
\langle z(t)\rangle=0, \quad B_{z}\left(t-t^{\prime}\right)=\left\langle z(t) z\left(t^{\prime}\right)\right\rangle=2 D \delta\left(t-t^{\prime}\right) .
$$

Решение этого уравнения имеет структуру

$$
y(t ; \alpha)=\exp \left\{-\alpha t+\int_{0}^{t} d \tau z(\tau)\right\} .
$$

Отметим, что изменение знака параметра $\alpha$ в (2) статистически эквивалентно переходу к процессу $1 / y(t)$.

На рис. 1 приведены реализации логнормального случайного процесса $y(t ; \alpha)$, заданного формулой (2), для положительного и отрицательного значений параметра $\alpha$ при $|\alpha| / D=1$ (штриховые линии соответствуют функциям $e^{-D t}$ при $\alpha>0$ и $e^{D t}$ при $\left.\alpha<0\right)$. Из этих рисунков видно наличие редких, но больших выбросов 


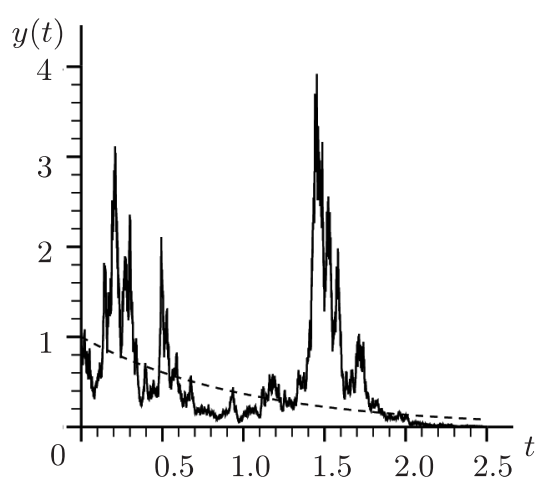

a

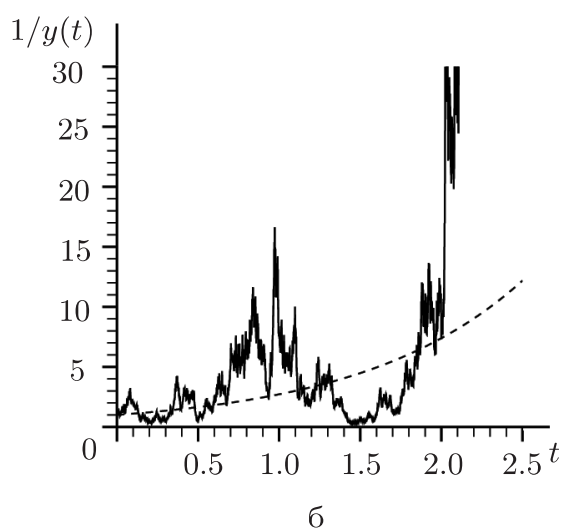

б

Рис. 1. Реализации логнормального процесса $y(t)$ при $\alpha>0$ (а) и $\alpha<0$ (б); здесь $|\alpha| / D=1$.

относительно штриховых линий как в сторону больших значений, так и к нулю. Такое свойство случайных процессов называется перемежаемостъю; оно интенсивно изучалось в 80-х гг. прошлого столетия, см., например, работы [13], [14]. Заслуга авторов этих работ состоит в том, что они, по-видимому, первыми обратили внимание на возможность стохастического структурообразования благодаря такому параметрическому возбуждению.

Под перемежаемостью случайного процесса в нашей работе подразумевается более или менее равномерное чередование выбросов этого процесса как в сторону бо́льших значений, так и в сторону меньших значений этого процесса относительно детерминированной кривой, которая является медианой интегральной функции распределения вероятностей и называется кривой типичной реализащии (см. п. 2.1). При этом особенностью логнормального случайного процесса является наличие редких больших выбросов процесса, обусловленное длинным пологим "хвостом" плотности вероятностей. Все традиционные статистические характеристики типа моментных и корреляционных функций любого порядка обусловлены этими выбросами.

Простейшей физической задачей такого типа является задача о диффузии частицы и системы частиц в случайном поле скоростей $\mathbf{u}(\mathbf{r}, t)$ с заданными статистическими свойствами в кинематическом приближении (см. монографии [15]--[19], где дана подробная библиография рассматриваемой проблемы). Эта задача описывается системой обыкновенных дифференциальных уравнений

$$
\frac{d}{d t} \mathbf{r}(t)=\mathbf{u}(\mathbf{r}(t), t), \quad \mathbf{r}(0)=\mathbf{r}_{0}
$$

Численное моделирование показывает, что динамика системы частиц существенно различается в зависимости от того, является ли случайное поле скоростей бездивергентным или дивергентным. Так, для конкретной реализации бездивергентного стационарного поля скоростей $\mathbf{u}(\mathbf{r})$ движущиеся частицы, в начальный момент времени равномерно расположенные в круге (в двумерном случае), лишь перемешиваются 
в области, ограниченной деформированным контуром. Возникает только сильная изрезанность этого контура, имеющая фрактальный характер. Для случая потенциального поля скоростей $\mathbf{u}(\mathbf{r})$ частицы, в начальный момент времени равномерно расположенные в квадрате, в процессе временно́й эволюции образуют кластерные области. Подчеркнем, что образование кластеров в этой задаче - чисто кинематический эффект. Очевидно, что при усреднении по ансамблю реализаций случайного поля скоростей такая особенность динамики частиц исчезает.

Имеют место также динамическая локализация Андерсона собственных функций одномерного стационарного уравнения Шредингера со случайным потенциалом [20] и, соответственно, динамическая локализация интенсивности волны в краевой задаче о волнах в случайных слоистых средах в части $\left[L_{0}, L\right]$ одномерного пространства [21]. Эта задача задается стохастическим уравнением Гельмгольца

$$
\frac{d^{2}}{d x^{2}} u(x)+k^{2}[1+\varepsilon(x)] u(x)=0
$$

с краевыми условиями непрерывности поля и его производной на границах слоя:

$$
u(L)+\left.\frac{i}{k} \frac{d u(x)}{d x}\right|_{x=L}=2, \quad u\left(L_{0}\right)-\left.\frac{i}{k} \frac{d u(x)}{d x}\right|_{x=L_{0}}=0 .
$$

Первой принципиальной особенностью стохастических динамических систем, описываемых уравнениями в частных производных, является тот факт, что их решения представляют собой случайные поля в пространстве и во времени. Сложность понимания этих процессов формирования структурообразования складывается из двух факторов. Во-первых, в каждой фиксированной точке пространства случайное поле является случайным процессом во времени. Во-вторых, для каждого фиксированного момента времени случайное поле есть случайный процесс по пространственной переменной. Именно для случайных процессов (по времени или по пространственной координате) и осуществляется перемежаемость (т. е. изменчивость), которая является общим свойством всех случайных процессов независимо от природы их происхождения.

Можно ввести простейшее обобщение логнормального случайного процесса (2) на логнормальное случайное поле по формуле

$$
f(\mathbf{r}, t ; \alpha)=f_{0}(\mathbf{r}) \exp \left\{-\alpha t+\int_{0}^{t} d \tau z(r, \tau)\right\}
$$

где $z(\mathbf{r}, t)$ - гауссово дельта-коррелированное во времени случайное поле с нулевым средним значением и корреляционной функцией

$$
B_{z}\left(\mathbf{r}-\mathbf{r}^{\prime}, t-t^{\prime}\right)=\left\langle z(\mathbf{r}, t) z\left(\mathbf{r}^{\prime}, t^{\prime}\right)\right\rangle=2 D\left(\mathbf{r}-\mathbf{r}^{\prime}\right) \delta\left(t-t^{\prime}\right) .
$$

Это поле удовлетворяет дифференциальному уравнению первого порядка

$$
\frac{d}{d t} f(\mathbf{r}, t ; \alpha)=\{-\alpha+z(\mathbf{r}, t)\} f(\mathbf{r}, t ; \alpha), \quad f(\mathbf{r}, 0 ; \alpha)=f_{0}(\mathbf{r}),
$$

параметрически зависящему от пространственной точки $\mathbf{r}$.

6 Теоретическая и математическая физика, т. 176, № 3, 2013 г. 
Отметим, что в упомянутых ранее работах по перемежаемости [13], [14] в качестве модельной задачи рассматривалось уравнение

$$
\frac{d}{d t} f(\mathbf{r}, t)=z(\mathbf{r}, t) f(\mathbf{r}, t)+\mu_{f} \Delta f(\mathbf{r}, t),
$$

где $\mu_{f}$ - динамический коэффициент диффузии для поля $f(\mathbf{r}, t)$. Это уравнение со случайным размножением и диффузией характерно как для задач биологии, так и для задач кинетики химических и ядерных реакций (см., например, работу [22]). При этом если $f_{0}(\mathbf{r})=1$, то все одноточечные статистические характеристики этого поля не зависят от $\mathbf{r}$.

На начальном этапе диффузии решением задачи (6) при $\alpha=0$ и $f_{0}(\mathbf{r})=1$ является функция (5)

$$
f(\mathbf{r}, t)=\exp \left\{\int_{0}^{t} d \tau z(\mathbf{r}, \tau)\right\}
$$

которая с точки зрения одноточечных статистических характеристик статистически эквивалентна случайному процессу $y(t ; 0)$, заданному в $(2)$.

Такое физическое явление, как кластеризация, в первую очередь возникает в задачах турбулентного переноса в случайном поле скоростей $\mathbf{u}(\mathbf{r}, t)$. В частности, может происходить кластеризация как пассивной скалярной примеси (поля плотности) [18], [19], [23], так и векторной примеси (энергии магнитного поля) в рамках кинематического приближения [18], [19], [24], [25]. Исходными стохастическими уравнениями при этом являются уравнение непрерывности для поля плотности примеси $\rho(\mathbf{r}, t)$,

$$
\left(\frac{\partial}{\partial t}+\frac{\partial}{\partial \mathbf{r}} \mathbf{u}(\mathbf{r}, t)\right) \rho(\mathbf{r}, t)=\mu_{\rho} \Delta \rho(\mathbf{r}, t), \quad \rho(\mathbf{r}, 0)=\rho_{0}(\mathbf{r}),
$$

и уравнение индукции для бездивергентного магнитного поля $\mathbf{H}(\mathbf{r}, t)[26]$,

$$
\begin{aligned}
\left(\frac{\partial}{\partial t}+\frac{\partial}{\partial \mathbf{r}} \mathbf{u}(\mathbf{r}, t)\right) \mathbf{H}(\mathbf{r}, t) & =\left(\mathbf{H}(\mathbf{r}, t) \cdot \frac{\partial}{\partial \mathbf{r}}\right) \mathbf{u}(\mathbf{r}, t)+\mu_{H} \Delta \mathbf{H}(\mathbf{r}, t), \\
\mathbf{H}(\mathbf{r}, 0) & =\mathbf{H}_{0}(\mathbf{r}),
\end{aligned}
$$

где $\mu_{\rho}$ и $\mu_{H}$ - динамические коэффициенты диффузии для поля плотности и магнитного поля. Здесь $\mathbf{u}(\mathbf{r}, t)$ - поле турбулентных скоростей, которое мы считаем однородным и изотропным в пространстве и стационарным во времени, с заданными статистическими свойствами.

Стохастическое структурообразование может осуществляться и в виде каустической структуры интенсивности волнового поля в задачах о волнах в случайно-неоднородных средах в рамках стохастического параболического уравнения Леонтовича (см., например, труды [18], [19], [27])

$$
\frac{\partial}{\partial x} u(x, \mathbf{R})=\frac{i}{2 k} \Delta_{\mathbf{R}} u(x, \mathbf{R})+\frac{i k}{2} \varepsilon(x, \mathbf{R}) u(x, \mathbf{R}), \quad u(x, \mathbf{R})=u_{0}(\mathbf{R})
$$

где $x$ - координата в направлении распространения волны, $\mathbf{R}$ - координаты в поперечной плоскости, a $\varepsilon(x, \mathbf{R})$ - отклонение диэлектрической проницаемости от единицы. Плоской падающей волне соответствует начальное условие $u(0, \mathbf{R})=u_{0}$. 
Отметим, что это же уравнение является нестационарным уравнением Шредингера со случайным потенциалом $\varepsilon(x, \mathbf{R})$ при замене $x$ на время $t$.

Если ввести амплитуду и фазу волнового поля по формуле

$$
u(x, \mathbf{R})=A(x, \mathbf{R}) e^{i S(x, \mathbf{R})},
$$

то уравнение для интенсивности волнового поля $I(x, \mathbf{R})=|u(x, \mathbf{R})|^{2}$ примет следующий вид:

$$
\frac{\partial}{\partial x} I(x, \mathbf{R})+\frac{1}{k} \nabla_{\mathbf{R}}\left\{\nabla_{\mathbf{R}} S(x, \mathbf{R}) I(x, \mathbf{R})\right\}=0, \quad I(0, \mathbf{R})=I_{0}(\mathbf{R}) .
$$

Это уравнение по форме совпадает с уравнением непрерывности (8) для поля плотности примеси в случайном потенциальном потоке при отсутствии динамического коэффициента диффузии.

Отметим также нелинейное обобщение уравнения (10), соответствующее монохроматической нелинейной задаче о самовоздействии волны в случайно-неоднородных средах, описываемой нелинейным параболическим уравнением Леонтовича (нелинейным уравнением Шредингера)

$$
\frac{\partial}{\partial x} u(x, \mathbf{R})=\frac{i}{2 k} \Delta_{\mathbf{R}} u(x, \mathbf{R})+\frac{i k}{2} \varepsilon(x, \mathbf{R} ; I(x, \mathbf{R})) u(x, \mathbf{R}), \quad u(0, \mathbf{R})=u_{0}(\mathbf{R}) .
$$

Для этого уравнения интенсивность волнового поля $I(x, \mathbf{R})$ также определяется уравнением (11), форма которого не зависит от вида функции $\varepsilon(x, \mathbf{R} ; I(x, \mathbf{R}))$.

Особенностью уравнений (8) и (9) является параметрическое возбуждение со временем в каждой реализации как поля плотности $\rho(\mathbf{r}, t)$ (для сжимаемого потока жидкости), так и энергии магнитного поля $E(\mathbf{r}, t)=\mathbf{H}^{2}(\mathbf{r}, t)$ (для турбулентного потока жидкости). Это называется стохастическим динамо (см., например, книгу [26]).

Такое параметрическое возбуждение сопровождается на начальных стадиях эволюции динамических систем возрастанием со временем всех традиционных статистических характеристик решения задачи типа моментных функций полей плотности $\left\langle\rho^{n}(\mathbf{r}, t)\right\rangle$ и энергии магнитного поля $\left\langle E^{n}(\mathbf{r}, t)\right\rangle$, а также их корреляционных функций любого порядка. Кроме того, имеет место возрастание с расстоянием моментов мощности излучения в случайных средах $\left\langle I^{n}(x, \mathbf{R})\right\rangle$.

На начальных этапах развития эффекты динамической диффузии для плотности и магнитного поля являются несущественными, и, пренебрегая ими, мы приходим к уравнениям в частных производных первого порядка:

$$
\begin{array}{rlrl}
\left(\frac{\partial}{\partial t}+\frac{\partial}{\partial \mathbf{r}} \mathbf{u}(\mathbf{r}, t)\right) \rho(\mathbf{r}, t) & =0, & \rho(\mathbf{r}, 0) & =\rho_{0}(\mathbf{r}), \\
\left(\frac{\partial}{\partial t}+\frac{\partial}{\partial \mathbf{r}} \mathbf{u}(\mathbf{r}, t)\right) \mathbf{H}(\mathbf{r}, t)=\left(\mathbf{H}(\mathbf{r}, t) \cdot \frac{\partial}{\partial \mathbf{r}}\right) \mathbf{u}(\mathbf{r}, t), & \mathbf{H}(\mathbf{r}, 0)=\mathbf{H}_{0}(\mathbf{r}) .
\end{array}
$$

Однако именно в этом интервале времени в отдельных реализациях соответствующих полей и могут зарождаться пространственные структурные образования!

Обнаружить и описать явление пространственного структурообразования (кластеризацию) в отдельных реализациях случайных полей удается только с помощью анализа одновременны́х и одноточечных плотностей вероятностей решений уравнений, приведенных выше, на основе идей статистической топографии. Рассмотрим сначала статистическое описание логнормальных случайных процессов. 


\section{2. ЛОГНОРМАЛЬНЫЕ ПРОЦЕССЫ И ДИНАМИЧЕСКАЯ ЛОКАЛИЗАЦИЯ}

Одновременна́я плотность вероятностей $P(t ; y)=\langle\delta(y(t)-y\rangle$ логнормального процесса (2) описывается уравнением Фоккера-Планка

$$
\begin{aligned}
\frac{\partial}{\partial t} P(y, t ; \alpha) & =\left(\alpha \frac{\partial}{\partial y} y+D \frac{\partial}{\partial y} y \frac{\partial}{\partial y} y\right) P(y, t ; \alpha), \\
P(y, 0 ; \alpha) & =\delta(y-1),
\end{aligned}
$$

решение которого, естественно, зависит от параметра $\alpha$ :

$$
P(y, t ; \alpha)=\frac{1}{2 y \sqrt{\pi D t}} \exp \left\{-\frac{\ln ^{2}\left(y e^{\alpha t}\right)}{4 D t}\right\} .
$$

Это распределение вероятностей имеет различный вид при $\alpha>0$ и $\alpha<0$. Общей особенностью для этих случаев является лишь появление длинных пологих "хвостов" при больших $t$, что означает усиление роли больших выбросов процесса $y(t ; \alpha)$ в формировании одновременно́й статистики.

Интегральная функция распределения вероятностей определяется выражением

$$
F(y, t ; \alpha)=\int_{-\infty}^{y} d y^{\prime} P\left(t ; y^{\prime}\right)=\mathrm{P}(y(t ; \alpha)<y)=\operatorname{Pr}\left(\frac{1}{\sqrt{2 D t}} \ln \left(y e^{\alpha t}\right)\right),
$$

где функция

$$
\operatorname{Pr}(z)=\frac{1}{\sqrt{2 \pi}} \int_{-\infty}^{z} d x e^{-x^{2} / 2}
$$

есть интеграл вероятностей. При этом очевидно, что $\operatorname{Pr}(\infty)=1$ и $\operatorname{Pr}(0)=1 / 2$. Исходя из выражения (13) легко написать асимптотику интеграла вероятностей при $z \rightarrow \pm \infty:$

$$
\left.\operatorname{Pr}(z)\right|_{z \rightarrow \infty} \approx 1-\frac{1}{z \sqrt{2 \pi}} e^{-z^{2} / 2},\left.\quad \operatorname{Pr}(z)\right|_{z \rightarrow-\infty} \approx \frac{1}{|z| \sqrt{2 \pi}} e^{-z^{2} / 2} .
$$

Также из уравнения (12) легко получить равенство

$$
\alpha=-\lim _{t \rightarrow \infty} \frac{\partial\langle\ln y(t ; \alpha)\rangle}{\partial t} .
$$

Следовательно, параметр $\alpha$ в уравнении (12) совпадает с ляпуновским характеристическим показателем (15) для логнормального случайного процесса $y(t ; \alpha)$, заданного в (2) (см., например, [20], [28], [29]).

2.1. Кривая типичной реализации случайного процесса. Статистические характеристики процесса $z(t)$ в фиксированный момент времени $t$ описываются его плотностью вероятностей $P(z, t)$ и интегральной функцией распределения вероятностей $F(Z, t)=\int_{-\infty}^{Z} d z^{\prime} P\left(z^{\prime}, t\right)$.

Кривой типичной реализации случайного процесса $z(t)$ называется детерминированная кривая $z^{*}(t)$, которая является медианой интегральной функции распределения вероятностей и определяется как решение алгебраического уравнения

$$
F\left(z^{*}(t), t\right)=\frac{1}{2} .
$$


Это означает, с одной стороны, что для любого момента времени $t$ имеет место равенство вероятностей $\mathrm{P}\left\{z(t)>z^{*}(t)\right\}=\mathrm{P}\left\{z(t)<z^{*}(t)\right\}=1 / 2$. С другой стороны, медиана имеет специфическое свойство, заключающееся в том, что для любого интервала $\left(t_{1}, t_{2}\right)$ случайный процесс $z(t)$ "обвивает" кривую $z^{*}(t)$ таким образом, что среднее время, в течение которого выполняется неравенство $z(t)>z^{*}(t)$, совпадает со средним временем, в течение которого выполняется обратное неравенство $z(t)<z^{*}(t)$

$$
\left\langle T_{z(t)>z^{*}(t)}\right\rangle=\left\langle T_{z(t)<z^{*}(t)}\right\rangle=\frac{1}{2}\left(t_{2}-t_{1}\right) .
$$

Кривая $z^{*}(t)$ может, конечно, существенно отличаться от любой конкретной реализации процесса $z(t)$ и не описывает величину возможных выбросов. Таким образом, кривая типичной реализации $z^{*}(t)$ случайного процесса $z(t)$, полученная с помощью одновременно́й плотности вероятностей, определена тем не менее на всем интервале времени $t \in(0, \infty)$ и является той детерминированной кривой, относительно которой осуществляется перемежаемость.

Кривая типичной реализации (16) для гауссова случайного процесса $z(t)$ совпадает со средним значением процесса $z(t)$, т. е. $z^{*}(t)=\langle z(t)\rangle$, а кривая типичной реализации для логнормального случайного процесса $f(t)=e^{z(t)}$ определяется равенством $f^{*}(t)=e^{\langle z(t)\rangle}=e^{\langle\ln f(t)\rangle}$. Следовательно, кривая типичной реализации логнормального процесса (2) задается как

$$
f^{*}(t)=e^{\langle\ln f(t)\rangle}=e^{-\alpha t}
$$

и совпадает с ляпуновской экспонентой. При $\alpha>0$ кривая типичной реализации экспоненциально убывает во времени, а в обратном случае $\alpha<0$ экспоненциально возрастает. Именно эти функции изображены на рис. 1 штриховыми линиями. При $\alpha=0$ перемежаемость осуществляется относительно прямой $f^{*}(t)=1$.

2.2. Динамическая локализация. Отметим, что для одномерных задач положительность ляпуновского характеристического индекса $\alpha$ как раз соответствует физическому явлению динамической локализации (кластеризации).

Так, для задачи диффузии системы частиц (3) в гауссовом потенциальном случайном поле скоростей $\mathbf{u}(\mathbf{r}, t)$ кривая типичной реализации для расстояния между двумя частицами есть экспоненциально убывающая функция времени. В более общем случае при наличии соленоидальной части спектра случайного поля скоростей знак параметра $\alpha$ зависит от соотношения между соленоидальной и потенциальной составляющими поля скоростей [15]-[19]. В несжимаемом потоке жидкости параметр $\alpha<0$, и частицы стремятся "разбежаться".

Для краевой задачи (4) о падении плоской волны на полупространство случайной слоистой среды интенсивность волнового поля $I(x)=|u(x)|^{2}$ представляет собой логнормальный случайный процесс, кривая типичной реализации для которого имеет вид $I^{*}(x)=2 e^{-D(L-x)}$, где для модели гауссова случайного процесса $\varepsilon(x)$ с корреляционной функцией $\left\langle\varepsilon(x) \varepsilon\left(x^{\prime}\right)\right\rangle=2 D \delta\left(x-x^{\prime}\right)$ параметр $D=k^{2} \sigma_{\varepsilon}^{2} l_{0} / 2$ (здесь $\sigma_{\varepsilon}^{2}$ - дисперсия процесса $\varepsilon(x)$, а $l_{0}$ - его корреляционный радиус). Коэффициент прохождения волны через достаточно толстый слой случайной среды для этой задачи экспоненциально убывает, и полупространство случайно-неоднородной среды 
$\left(L_{0} \rightarrow-\infty\right)$ полностью отражает падающую на него волну. В этом случае интенсивность волнового поля $I(x)$ статистически эквивалентна случайному процессу $2 y(t ; \alpha)$ при $\alpha=D$, и ее реализация похожа на зеркальное отражение рис. 1а. При этом, разумеется, имеет место экспоненциальное возрастание моментов интенсивности волнового поля в глубь среды от источника.

В монографии [20] указанный эффект был установлен на основе анализа ляпуновской экспоненты для этой задачи, для логнормального процесса ляпуновская экспонента совпадает с кривой типичной реализации. Отметим также, что для этой задачи величина, обратная к коэффициенту диффузии $D$, определяющая естественный масштаб длины, который связан со случайными неоднородностями среды, обычно называется длиной локализации, т. е. $l_{\mathrm{loc}}=1 / D$.

\section{3. ЛОГНОРМАЛЬНЫЕ ПОЛЯ И КЛАСТЕРИЗАЦИЯ}

3.1. Случайные логнормальные поля. Рассмотрим положительное логнормальное случайное поле $f(\mathbf{r}, t)$, одноточечная плотность вероятностей которого

$$
P(\mathbf{r}, t ; f)=\langle\delta(f(\mathbf{r}, t)-f)\rangle
$$

задается уравнением

$$
\frac{\partial}{\partial t} P(\mathbf{r}, t ; f)=\left\{D_{0} \frac{\partial^{2}}{\partial \mathbf{r}^{2}}+\alpha \frac{\partial}{\partial f} f+D_{f} \frac{\partial}{\partial f} f \frac{\partial}{\partial f} f\right\} P(\mathbf{r}, t ; f)
$$

с начальным условием $P(\mathbf{r}, 0 ; f)=\delta\left(f-f_{0}(\mathbf{r})\right)$, где $D_{0}-$ коэффициент диффузии в r-пространстве, а коэффициенты $\alpha$ и $D_{f}$ характеризуют диффузию в $f$-пространстве. При этом параметр $\alpha$ может быть как положительным, так и отрицательным или равным нулю (критический случай). Изменение знака $\alpha$ для одноточечных характеристик означает переход от поля $f(\mathbf{r}, t)$ к полю $\tilde{f}(\mathbf{r}, t)=1 / f(\mathbf{r}, t)$.

Решение уравнения (17) имеет вид

$$
P(\mathbf{r}, t ; f)=\frac{1}{2 f \sqrt{\pi D_{f} t}} \exp \left\{D_{0} t \frac{\partial^{2}}{\partial \mathbf{r}^{2}}\right\} \exp \left\{-\frac{\ln ^{2}\left[f e^{\alpha t} / f_{0}(\mathbf{r})\right]}{4 D_{f} t}\right\} .
$$

Для положительного консервативного случайного поля $f(\mathbf{r}, t)$, для которого

$$
\int d \mathbf{r} f(\mathbf{r}, t)=\int d \mathbf{r} f_{0}(\mathbf{r})
$$

имеем $\alpha=D_{f}$, и уравнение (17) можно переписать в виде

$$
\frac{\partial}{\partial t} P(\mathbf{r}, t ; f)=\left(D_{0} \frac{\partial^{2}}{\partial \mathbf{r}^{2}}+\alpha \frac{\partial^{2}}{\partial f^{2}} f^{2}\right) P(\mathbf{r}, t ; f) .
$$

Разумеется, для любого случайного поля $f(\mathbf{r}, t)$ также всегда имеет место свойство перемежаемости. Прежде всего, для любой фиксированной пространственной точки $\mathbf{r}$ эволюция во времени $f(\mathbf{r}, t)$ есть случайный процесс, для которого справедливо все сказанное выше. 
Для статистически пространственно-однородной задачи, которая соответствует начальному распределению поля $f_{0}(\mathbf{r})=f_{0}$, все одноточечные статистические характеристики поля $f(\mathbf{r}, t)$ не зависят от точки $\mathbf{r}$, и положительность индекса

$$
\alpha=-\lim _{t \rightarrow \infty} \frac{\partial\langle\ln f(\mathbf{r}, t)\rangle}{\partial t}
$$

для логнормального поля $f(\mathbf{r}, t)$ означает, что в любой точке пространства реализации этого поля убывают во времени, невзирая на то что в логнормальном процессе присутствуют большие редкие выбросы. При этом характерное время убывания поля $t \sim 1 / \alpha$. Если это поле почти всюду убывает, то где-то оно должно и концентрироваться, т. е. должна быть кластеризация. При отрицательном значении параметра $\alpha$ происходит рост поля в каждой фиксированной точке пространства. В этом случае плотность вероятностей (18) не зависит от $\mathbf{r}$ и описывается уравнением

$$
\frac{\partial}{\partial t} P(t ; f)=\left\{\alpha \frac{\partial}{\partial f} f+D_{f} \frac{\partial}{\partial f} f \frac{\partial}{\partial f} f\right\} P(t ; f), \quad P(0 ; f)=\delta\left(f-f_{0}\right),
$$

решение которого дается формулой

$$
P(t ; f)=\frac{1}{2 f \sqrt{\pi D_{f} t}} \exp \left\{-\frac{\ln ^{2}\left[f e^{\alpha t} / f_{0}\right]}{4 D_{f} t}\right\} .
$$

Таким образом, для пространственно-однородной задачи одноточечные статистические характеристики случайного поля $f(\mathbf{r}, t)$ статистически эквивалентны статистическим характеристикам логнормального процесса $f(t ; \alpha)$ с плотностью вероятностей (20). Характерной особенностью этого распределения является появление длинного пологого "хвоста" при $D_{f} t \gg 1$, что означает усиление роли больших выбросов процесса $f(t ; \alpha)$ в формировании одновременно́й статистики. Для этого распределения все моментные функции любого порядка $n$ экспоненциально возрастают во времени. В частности, при $n=1$ и $D_{f}>\alpha$ среднее значение задается формулой

$$
\langle f(\mathbf{r}, t)\rangle=f_{0} e^{\left(D_{f}-\alpha\right) t}
$$

а величина $\alpha$ является ляпуновским характеристическим индексом.

Описать пространственную кластеризацию случайного поля $f(\mathbf{r}, t)$ в почти каждой реализации этого поля можно на основе идей статистической топографии (см. , например, монографии [15]-[19], где приведены библиографические ссылки по этому вопросу).

3.2. Статистическая топография случайных полей. В статистической топографии случайных полей основным объектом изучения, как и в обычной топографии горных массивов, является система контуров - линий уровня (в двумерном случае) или поверхностей (в трехмерном случае) постоянных значений, определяемых равенством $f(\mathbf{r}, t)=f=$ const. Для анализа системы контуров (для простоты в данном пункте ограничимся двумерным случаем $\mathbf{r}=\mathbf{R}$ ) удобно ввести сосредоточенную на этих контурах дельта-функцию Дирака

$$
\varphi(\mathbf{R}, t ; f)=\delta(f(\mathbf{R}, t)-f),
$$


называемую индикаторной функиией. Через эту функцию выражаются, например, такие величины, как общая площадь, ограниченная линиями уровня областей, в которых случайное поле $f(\mathbf{R}, t)$ превышает заданный уровень $f$, т. е. $f(\mathbf{R}, t)>f$,

$$
S(t ; f)=\int d \mathbf{R} \theta(f(\mathbf{R}, t)-f)=\int d \mathbf{R} \int_{f}^{\infty} d f^{\prime} \varphi\left(\mathbf{R}, t ; f^{\prime}\right),
$$

и общая "масса" поля, заключенная в этих областях,

$$
M(t ; f)=\int d \mathbf{R} f(\mathbf{R}, t) \theta(f(\mathbf{R}, t)-f)=\int d \mathbf{R} \int_{f}^{\infty} d f^{\prime} f^{\prime} \varphi\left(\mathbf{R}, t ; f^{\prime}\right),
$$

где $\theta(f(\mathbf{R}, t)-f)$ - тета-функция Хевисайда.

Среднее значение индикаторной функции (21) по ансамблю реализаций случайного поля $f(\mathbf{R}, t)$ определяет одновременну́ю и одноточечную плотность вероятностей

$$
P(\mathbf{R}, t ; f)=\langle\delta(f(\mathbf{R}, t)-f)\rangle,
$$

следовательно, средние по ансамблю реализаций значения величин $S(t ; f)$ и $M(t ; f)$ определяются непосредственно этой плотностью вероятностей:

$$
\langle S(t ; f)\rangle=\int d \mathbf{R} \int_{f}^{\infty} d f^{\prime} P\left(\mathbf{R}, t ; f^{\prime}\right), \quad\langle M(t ; f)\rangle=\int d \mathbf{R} \int_{f}^{\infty} d f^{\prime} f^{\prime} P\left(\mathbf{R}, t ; f^{\prime}\right) .
$$

Дополнительную информацию о детальной структуре поля $f(\mathbf{R}, t)$ можно получить, включив в рассмотрение его пространственный градиент $\mathbf{p}(\mathbf{R}, t)=\boldsymbol{\nabla} f(\mathbf{R}, t)$. Так, например, величина

$$
l(t ; f)=\int d \mathbf{R}|\mathbf{p}(\mathbf{R}, t)| \delta(f(\mathbf{R}, t)-f)=\oint d l
$$

описывает общую длину контуров. Подынтегральное выражение в (23) определяется расширенной индикаторной функцией

$$
\varphi(\mathbf{R}, t ; f, \mathbf{p})=\delta(f(\mathbf{R}, t)-f) \delta(\mathbf{p}(\mathbf{R}, t)-\mathbf{p}),
$$

и среднее значение $l(t ; f)$ (см. формулу $(23))$ связано с совместной одновременно́й плотностью вероятностей поля $f(\mathbf{R}, t)$ и его градиента $\mathbf{p}(\mathbf{R}, t)$, которая определятся усреднением индикаторной функции (24) по ансамблю реализаций, т. е. функцией

$$
P(\mathbf{R}, t ; f, \mathbf{p})=\langle\delta(f(\mathbf{R}, t)-f \delta(\mathbf{p}(\mathbf{R}, t)-\mathbf{p})\rangle .
$$

Включение в рассмотрение пространственных производных второго порядка позволяет оценить общее число контуров $f(\mathbf{R}, t)=f=$ const с помощью приближенной (с точностью до незамкнутых линий) формулы

$$
\begin{aligned}
N(t ; f) & =N_{\text {in }}(t ; f)-N_{\text {out }}(t ; f)= \\
& =\frac{1}{2 \pi} \int d \mathbf{R} \kappa(t, \mathbf{R} ; f)|\mathbf{p}(\mathbf{R}, t)| \delta(f(\mathbf{R}, t)-f),
\end{aligned}
$$

где $N_{\text {in }}(t ; f)$ и $N_{\text {out }}(t ; f)$ - числа контуров, для которых вектор $\mathbf{p}$ направлен по внутренней и внешней нормалям соответственно, $\kappa(t, \mathbf{R} ; f)$ - кривизна линии уровня. 
3.2.1. Условия возникновения кластерных структур. Обсудим условия возникновения стохастического структурообразования для параметрически возбуждаемых случайных полей. Ясно, что для положительного поля $f(\mathbf{R}, t)$ в общем случае условием его кластеризации с вероятностью единица, т. е. почти для всех его реализаций, является следующая тенденция поведения средних величин в пределе $t \rightarrow \infty$ :

$$
\langle S(t ; f)\rangle \rightarrow 0, \quad\langle M(t ; f)\rangle \rightarrow \int d \mathbf{R}\langle f(\mathbf{R}, t)\rangle .
$$

Отсутствию структурообразования отвечает тенденция поведения в пределе $t \rightarrow \infty$ следующего вида:

$$
\langle S(t ; f)\rangle \rightarrow \infty, \quad\langle M(t ; f)\rangle \rightarrow \int d \mathbf{R}\langle f(\mathbf{R}, t)\rangle .
$$

Для пространственно-однородного поля $f(\mathbf{R}, t)$ одноточечная плотность вероятностей $P(\mathbf{R}, t ; f)$ не зависит от $\mathbf{R}$. В этом случае статистические средние всех выражений (без интегрирования по $\mathbf{R}$ ) определяют удельные (приходящиеся на единицу площади) значения этих величин. Так, удельная средняя площадь $\left\langle s_{\mathrm{hom}}(t ; f)\right\rangle$ области, в которой случайное поле $f(\mathbf{R}, t)$ превышает заданный уровень $f$, совпадает с вероятностью события $f(\mathbf{R}, t)>f$ в любой точке пространства,

$$
\left\langle s_{\text {hom }}(t ; f)\right\rangle=\langle\theta(f(\mathbf{R}, t)-f)\rangle=\mathrm{P}\{f(\mathbf{R}, t)>f\},
$$

и средняя удельная площадь дает геометрическую интерпретациию вероятности события $f(\mathbf{R}, t)>f$, не зависящей, разумеется, от точки $\mathbf{R}$. Таким образом, условия кластеризации для однородного случая сводятся к следующей тенденции поведения средних величин в пределе $t \rightarrow \infty$ :

$$
\left\langle s_{\text {hom }}(t ; f)\right\rangle=\mathrm{P}\{f(\mathbf{r}, t)>f\} \rightarrow 0, \quad\left\langle m_{\text {hom }}(t ; f)\right\rangle \rightarrow\langle f(t)\rangle .
$$

Отсутствию кластеризации соответствует тенденция поведения в пределе $t \rightarrow \infty$ следующего вида:

$$
\left\langle s_{\text {hom }}(t ; f)\right\rangle=\mathrm{P}\{f(\mathbf{r}, t)>f\} \rightarrow 1, \quad\left\langle m_{\text {hom }}(t ; f)\right\rangle \rightarrow\langle f(t)\rangle .
$$

Подведем итог. Кластеризачия в пространственно-однородной задаче есть явление, происходящее с вероятностью единища (т.е. почти для всех реализаций случайного положительного поля), порожденное редким событием, вероятность которого стремится $к$ нулю.

В данном случае само наличие редких событий является тем "спусковым механизмом", который запускает процесс структурообразования, а само структурообразование есть свойство случайной среды, что, по своей сути, является законом природы [30], [31]. При этом характерное время образования кластерной структуры в пространстве определяется характером приведенных выше асимптотических выражений при больших временах. Это время теперь зависит не только от статистического ляпуновского характеристического индекса $\alpha$, но и от коэффициента диффузии $D_{f}$ в фазовом пространстве положительного поля $f(\mathbf{r}, t)$. Конечно, это 


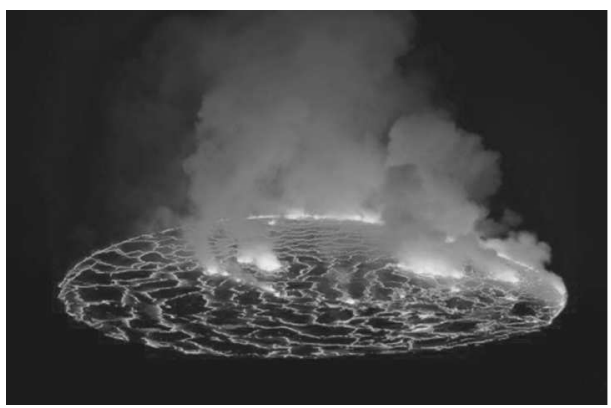

a

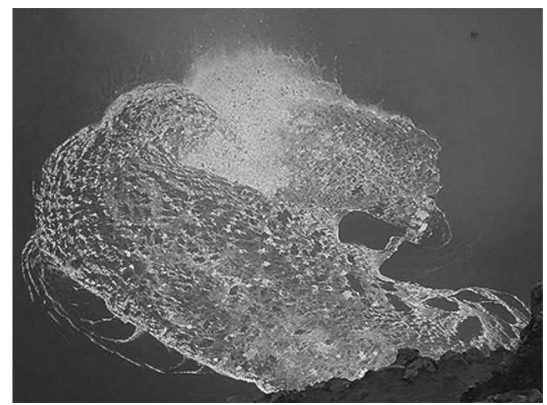

б

Рис. 2. Озеро кипящей лавы в кратере вулкана Нирагонго в районе Великих озер в Африке (а) и кратер Халемаумау вулкана Килауэа в Гавайском вулканическом национальном парке (б).

время больше, чем характерное время убывания реализаций в каждой фиксированной точке пространства.

Для конкретных физических динамических систем задача о кластеризации физических полей сводится, таким образом, к вычислению стохастического ляпуновского индекса $\alpha$ и коэффициента диффузии $D_{f}$, что, вообе говоря, является довольно громоздкой задачей для конкретных уравнений в частных производных. При наличии кластеризации поле в большей части пространства просто отсутствует! Разумеется, приведенные выше условия наличия и отсутствия явления кластеризации поля $f(\mathbf{R}, t)$ не имеют никакого отношения к параметрическому росту во времени статистических характеристик типа моментных и корреляционных функций любого порядка.

Сформулированный критерий "идеальной кластеризации" (по аналогии с идеальной жидкостью) определяет динамику образования кластеров для динамических систем, описываемых, вообще говоря, уравнениями в частных производных первого порядка. Эта идеальная структура реализуется в виде очень тонкой ленты (в двумерном случае) или очень тонких трубок (в трехмерном случае). Отметим, что уравнения в частных производных первого порядка могут быть решены в общем случае с помощью метода характеристик. Это соответствует лагранжевому описанию динамических систем. При этом характеристические кривые, которые описываются обыкновенными дифференциальными уравнениями, могут, разумеется, иметь различные особенности и даже сингулярности. Условия возникновения таких особенностей в лагранжевом описании не имеют прямой связи с явлением кластеризации в пространстве и времени, т. е. в эйлеровом описании.

Для реальных физических систем в дальнейшем начинают проявляться различные дополнительные факторы, связанные с генерацией пространственных производных случайного поля, которые деформируют, но не уничтожают эту картину кластеризации. В частности, возможна ситуация, когда соответствующая плотность вероятностей выходит на стационарный режим $P(\mathbf{R} ; f)$ при $t \rightarrow \infty$. В этом случае функционалы типа (22) уже не описывают дальнейшую деформацию кластерной 


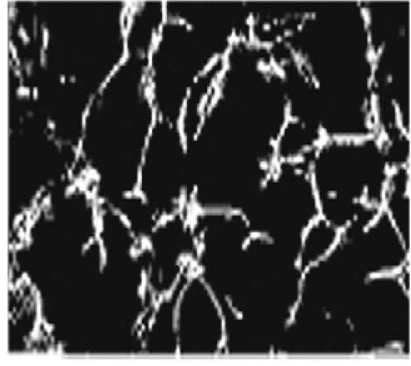

a

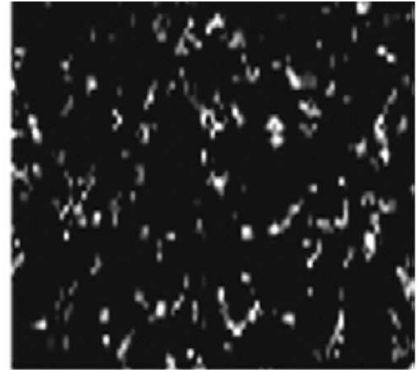

6

Рис. 3. Поперечное сечение лазерного пучка при его распространении в турбулентной среде в области сильных фокусировок (а) и в области сильных (насыщенных) флуктуаций (б): экспериментальные данные.

картины, и необходимо изучать временну́ю эволюцию функционалов, связанных с пространственными производными поля $f(\mathbf{R}, t)$ типа общей длины контуров и числа контуров.

В качестве примеров "идеальной" и “деформируемой" кластеризации в природе можно указать на озера лавы в кратерах вулканов ${ }^{1)}$ (см. рис. 2). При этом отметим, что статистической теории описания динамики вулканической лавы, насколько нам известно, в настоящее время не существует.

Подобную структуру имеют и изображенные на рис. 3, 4 явления, которые связаны с параболическим уравнением Леонтовича (10). Полное решение задачи о вычислении статистических характеристик решения уравнения (10) при $x \rightarrow \infty$ было осуществлено в работе [32] на основе записи решения этого уравнения в виде континуального интеграла.

Для малых расстояний, проходимых плоской падающей волной, распределение вероятностей интенсивности волнового поля, очевидно, имеет логнормальный характер, и в этом случае начинает происходить стохастическое структурообразование (кластеризация). При увеличении расстояния необходимо принимать во внимание нелинейный характер уравнения для комплексной фазы. Эта область флуктуаций, называемая областью сильных фокусировок, очень трудна для аналитических исследований. При еще бо́льших расстояниях, проходимых волной, статистические характеристики интенсивности выходят на режим насыщения, и эта пространственная область называется областью сильных флуктуаций интенсивности. В этой области статистические характеристики интенсивности волнового поля перестают зависеть от расстояния и принимают вид (при $u_{0}=1$ )

$$
\left\langle I^{n}(x, \mathbf{R})\right\rangle=n !, \quad P(x, I)=e^{-I} .
$$

С точки зрения статистической топографии средняя удельная площадь областей, внутри которых $I(x, \mathbf{R})>I$, и средняя удельная мощность, сосредоточенная в них,

1) Приведенные на рис. 2 изображения можно найти по адресам http://bigpicture.ru/?p=128340 и http://pacificislandparks.com/2010/01/20/more-amazing-lava-lake-photos/. 


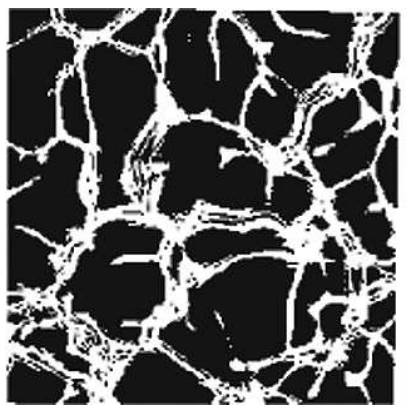

a

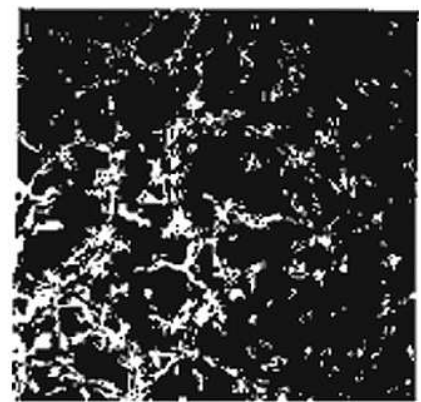

б

Рис. 4. То же что, на рис. 3: численное моделирование.

являются постоянными и при $x \rightarrow \infty$ не описывают поведение интенсивности волнового поля в отдельных реализациях. Кроме того, в этом случае не информативен и переход к статистически эквивалентному случайному процессу. Объяснить структуру волнового поля в отдельных реализациях в этом случае удалось на основе анализа таких величин, как удельная средняя длина контуров и удельное среднее число контуров интенсивности волнового поля, описываемых функционалами типа (23) и $(25)$ и связанных с пространственными производными интенсивности волнового поля (см. труды [15]-[19]). Эти функции продолжают возрастать с возрастанием расстояния и в области сильных флуктуаций интенсивности, следовательно, происходит дробление контуров, что наблюдалось как в лабораторных экспериментах, так и при численном моделировании.

3.2.2. Статистическая топография логнормальных случайных полей. При анализе одноточечных статистических характеристик пространственно-однородной задачи в общем случае удобно учитывать, что случайное поле $f(\mathbf{R}, t)$ статистически эквивалентно некоему случайному процессу $f(t)$ с теми же статистическими характеристиками.

Зная одноточечную плотность вероятностей случайного поля $f(\mathbf{r}, t)$, заданного формулой (18), можно получить также общую информацию о пространственной структуре случайного поля $f(\mathbf{r}, t)$. В частности, такие функционалы случайного поля $f(\mathbf{r}, t)$, как общая средняя величина объема (в трехмерном случае) или площади (в двумерном случае) области, в которой $f(\mathbf{r}, t)>f$, и общая средняя "масса" поля, заключенная в этой области, задаются равенствами

$$
\langle V(t, f)\rangle=\int d \mathbf{r} \int_{f}^{\infty} d f^{\prime} P\left(\mathbf{r}, t ; f^{\prime}\right), \quad\langle M(t, f)\rangle=\int d \mathbf{r} \int_{f}^{\infty} d f^{\prime} f^{\prime} P\left(\mathbf{r}, t ; f^{\prime}\right) .
$$

Значения этих функционалов не зависят от диффузии в r-пространстве (от коэффициента $\left.D_{0}\right)$ и в случае распределения вероятностей (18) получаем выражения

$$
\begin{aligned}
\langle V(t, f)\rangle & =\int d \mathbf{r} \operatorname{Pr}\left(\frac{1}{\sqrt{2 D t}} \ln \left(\frac{f_{0}(\mathbf{r})}{f} e^{-\alpha t}\right)\right) \\
\langle M(t, f)\rangle & =e^{(D-\alpha) t} \int d \mathbf{r} f_{0}(\mathbf{r}) \operatorname{Pr}\left(\frac{1}{\sqrt{2 D t}} \ln \left(\frac{f_{0}(\mathbf{r})}{f} e^{(2 D-\alpha) t}\right)\right),
\end{aligned}
$$

где интеграл вероятностей $\operatorname{Pr}(z)$ определяется равенством (13). 
Учитывая теперь асимптотики (14) функции $\operatorname{Pr}(z)$, можно изучить эволюцию этих функционалов во времени. А именно, асимптотика среднего объема при $t \rightarrow \infty$ убывает во времени при $\alpha>0$ по закону

$$
\langle V(t, f)\rangle \approx \frac{1}{\alpha} \sqrt{\frac{D}{\pi f^{\alpha / D} t}} e^{-\alpha^{2} t / 4 D} \int d \mathbf{r} \sqrt{f_{0}^{\alpha / D}(\mathbf{r})} .
$$

При $\alpha<0$ средний объем при $t \rightarrow \infty$ занимает все пространство.

Для полной средней "массы" получаем (в наиболее интересном случае $\alpha<2 D$ ) при $t \rightarrow \infty$ асимптотику вида

$$
\begin{aligned}
\langle M(t, f)\rangle \approx & e^{(D-\alpha) t} \int d \mathbf{r} f_{0}(\mathbf{r}) \times \\
& \times\left[1-\frac{1}{2 D-\alpha} \sqrt{\frac{D}{\pi t}\left(\frac{f}{f_{0}(\mathbf{r})}\right)^{(2 D-\alpha) / D}} e^{-(2 D-\alpha)^{2} t / 4}\right] .
\end{aligned}
$$

Следовательно, при $\alpha>0$ в кластерах в пределе $t \rightarrow \infty$ содержится вся средняя "масса".

Для однородных начальных условий соответствующие выражения без интегрирования по $\mathbf{r}$ описывают удельные значения объема больших выбросов и их общей "массы", приходящиеся на единицу объема:

$$
\begin{aligned}
\left\langle v_{\text {hom }}(t, f)\right\rangle & =\langle\theta(f(\mathbf{r}, t)-f)\rangle=\operatorname{P}\{f(\mathbf{r}, t)>f\}=\operatorname{Pr}\left(\frac{1}{\sqrt{2 D t}} \ln \left(\frac{f_{0}}{f} e^{-\alpha t}\right)\right), \\
\left\langle m_{\text {hom }}(t, f)\right\rangle & =f_{0} e^{(D-\alpha) t} \operatorname{Pr}\left(\frac{1}{\sqrt{2 D t}} \ln \left(\frac{f_{0}}{f} e^{(2 D-\alpha) t}\right)\right) .
\end{aligned}
$$

Если выбрать уровень сечения $f>f_{0}$, то в начальный момент времени мы получаем $\left\langle v_{\text {hom }}(0, f)\right\rangle=0$ и $\left\langle m_{\text {hom }}(0, f)\right\rangle=0$. Далее возникают пространственные возмущения случайного поля $f(\mathbf{r}, t)$, и при $t \rightarrow \infty$ мы имеем асимптотические выражения (в случае $2 D>\alpha$ )

$$
\begin{aligned}
& \left\langle v_{\text {hom }}(t, f)\right\rangle \approx \begin{cases}\frac{1}{\alpha} \sqrt{\frac{D}{\pi t}\left(\frac{f_{0}}{f}\right)^{\alpha / D}} e^{-\alpha^{2} t / 4 D}, & \alpha>0, \\
1-\frac{1}{|\alpha|} \sqrt{\frac{D}{\pi t}\left(\frac{f}{f_{0}}\right)^{|\alpha| / D}} e^{-\alpha^{2} t / 4 D}, & \alpha<0,\end{cases} \\
& \left\langle m_{\text {hom }}(t, f)\right\rangle \approx f_{0} e^{(D-\alpha) t}\left[1-\frac{1}{(2 D-\alpha)} \sqrt{\frac{D}{\pi t}\left(\frac{f}{f_{0}}\right)^{(2 D-\alpha) / D}} e^{-(2 D-\alpha)^{2} t / 4 D}\right] .
\end{aligned}
$$

Таким образом, при $\alpha>0$ удельный общий объем стремится к нулю, а удельная общая "масса", заключенная в этом объеме, стремится к средней "массе" всего пространства, что соответствует критерию структурообразования с вероятностью единица для "идеальной кластеризации" рассматриваемого поля $f(\mathbf{r}, t)$. При этом случайное поле $f(\mathbf{r}, t)$ практически отсутствует в большей части пространства. В данном случае в каждой фиксированной точке пространства характерное время убывания поля $\alpha t \sim 1$, а характерное время образования кластерной структуры поля $\alpha t \sim \max \left\{4 \xi, 4 \xi /(2 \xi-1)^{2}\right\}$, где $\xi=D / \alpha$. 
При $\alpha<0$ кластеризация отсутствует, происходит лишь общее усиление случайного поля $f(\mathbf{r}, t)$ во всем пространстве. Таким образом, в этом случае хаос остается хаосом! Происходит лишь кластеризация нулей поля $f(\mathbf{r}, t)$.

Отметим, что в критическом случае $\alpha=0$ средний удельный объем стремится при $t \rightarrow \infty$ к $1 / 2$, и в нем сосредоточена вся удельная средняя масса. При этом случайное поле $f(\mathbf{r}, t)$, заданное формулой $(7)$, статистически эквивалентно случайным процессам $f(t ; 0)$ и $1 / f(t ; 0)$, и кластеризация не осуществляется - имеется лишь общее свойство перемежаемости. Именно такая модель случайного поля $f(\mathbf{r}, t)$ рассматривалась в упомянутых выше работах [13], [14] по перемежаемости.

Имеет место следующая теорема.

Теорема 1. В статистически однородной задаче консервативное положительное параметрически возбуждаемое случайное логнормальное поле всегда кластеризуется с вероятностью единица, т.е. почти для всех реализаций этого поля.

В самом деле, для этого случая $f(\mathbf{r}, t)=e^{\ln f(\mathbf{r}, t)}$, следовательно,

$$
\langle f(\mathbf{r}, t)\rangle=\left\langle e^{\ln f(\mathbf{r}, t)}\right\rangle=\exp \left\{\langle\ln f(\mathbf{r}, t)\rangle+\frac{1}{2} \sigma_{\ln f(\mathbf{r}, t)}^{2}\right\},
$$

где $\sigma_{\ln f(\mathbf{r}, t)}^{2}-$ дисперсия случайного поля $\ln f(\mathbf{r}, t)$. Учитывая теперь, что в силу консервативности

$$
\langle\ln f(\mathbf{r}, t)\rangle+\frac{1}{2} \sigma_{\ln f(\mathbf{r}, t)}^{2}=\ln f_{0},
$$

получаем для кривой типичной реализации выражение

$$
f^{*}(\mathbf{r}, t)=e^{\langle\ln f(\mathbf{r}, t)\rangle}=f_{0} e^{-\alpha t}
$$

где ляпуновский характеристический параметр

$$
\alpha=\lim _{t \rightarrow \infty} \frac{1}{2 t} \sigma_{\ln f(\mathbf{r}, t)}^{2}>0 .
$$

Задача состоит в его вычислении из соответствующего динамического уравнения. При этом, как указывалось выше, $\alpha=D$ для консервативного поля $f(\mathbf{r}, t)$ (см. уравнение (19)). Следовательно, характерное время образования кластерной структуры $\alpha t \sim 4$, что в четыре раза превышает характерное время убывания поля в почти каждой точке пространства.

\section{4. ЗАКЛЮЧЕНИЕ}

Мы рассмотрели процесс стохастического структурообразования в динамических системах с параметрическим возбуждением положительных случайных полей $f(\mathbf{r}, t)$, которые описываются уравнениями в частных производных. Такое структурообразование в пространстве и во времени либо есть, либо его нет! А если оно осуществляется в пространстве, то это происходит в конкретных реализациях почти всегда, т. е. с вероятностью единица (экспоненциально быстрым образом), и состоит для пространственной однородной статистической задачи в следующем:

1) с течением времени поле почти во всех точках пространства убывает (разумеется, с какими-то флуктуациями); 
2) в само́м пространстве возникают области малого объема, где это поле концентрируется (кластеризуется), и стохастическое структурообразование обусловлено диффузией случайного поля $f(\mathbf{r}, t)$ в своем фазовом пространстве $\{f\}$.

В рассматриваемом случае кластеризация поля $f(\mathbf{r}, t)$ любой природы является общим свойством динамических полей, и можно сказать, что структурообразование для любых таких случайных полей - закон природы.

В работе выяснены условия, при которых такое структурообразование осуществляется. Примечательно, что эти условия имеют прозрачный физико-математический смысл и описываются на достаточно элементарном математическом уровне на основе идей статистической топографии.

Благодарности. Автор признателен рецензенту за моральную поддержку и полностью согласен с его замечанием, что "парадоксальность ситуации состоит в том, что многие конкретные результаты, обсуждаемые в статье, так или иначе возникали в предыдущих работах различных авторов, тем не менее они очень плохо усваиваются физической общественностью". Работа выполнена при поддержке РФФИ (грант № 13-05-0004) и Государственного контракта № 3/ГФ/Н-08.

\section{Список литературы}

[1] И. Пригожин, Г. Николис, Познание сложного. Введение, УРСС, М., 2008.

[2] В. И. Кляцкин, ПММ, 33:5 (1969), 889-891.

[3] В. И. Кляцкин, Изв. РАН. Физ. атм. и океана, 31:6 (1995), 749-754.

[4] V. Klyatskin, D. Gurarie, Phys. D, 98:2-4 (1996), 466-480.

[5] H. J. Hopfinger, F. K. Browand, Nature, 295 (1982), 393-394.

[6] R. W. Griffiths, E. J. Hopfinger, Deep Sea Research A, 31:3 (1984), 245-269.

[7] E. J. Hopfinger, "Turbulence and vortices in rotating fluids", Theoretical and Applied Mechanics (Saint-Martin-d'Heres, France, August 21-27, 1988), eds. P. Germain, M. Piau, D. Caillerie, North-Holland, Amsterdam, 1989, 117-138.

[8] B. M. Boubnov, G.S. Golitsyn, J. Fluid Mech., 167:6 (1986), 503-531.

[9] B. M. Boubnov, G.S. Golitsyn, Convection in Rotating Fluids, Fluid Mechanics and its Applications, 29, Kluwer, Dordrecht, 1995.

[10] С. С. Каримова, О. Ю. Лаврова, Д. М. Соловьев, Исследование Земли из космоса, 5 (2011), 15-23.

[11] S. Karimova, Adv. Space Res., 50:8 (2012), 1107-1124.

[12] Г. С. Голицын, Статистика и динамика природных процессов и явлений: методы, инструментарий, результаты, УРСС, М., 2013.

[13] Я. Б. Зельдович, С. А. Молчанов, А. А. Рузмайкин, Д. Д. Соколов, ЖЭТФ, 89:6 (1985), 2061-2072.

[14] Я. Б. Зельдович, С. А. Молчанов, А. А. Рузмайкин, Д. Д. Соколов, УФН, 152:5 (1987), $3-32$.

[15] В.И. Кляцкин, Стохастические уравнения глазами физика. Основные положения, точные результаты и асимптотические приближения, Физматлит, М., 2001.

[16] В. И. Кляцкин, Динамика стохастических систем, Физматлит, М., 2002.

[17] В. И. Кляцкин, Стохастические уравнения. Теория и ее приложения к акустике, гидродинамике и радиофизике, Физматлит, М., 2008.

[18] V. I. Klyatskin, Lectures on Dynamics of Stochastic Systems, Elsevier, Amsterdam, 2010.

[19] В. И. Кляцкин, Очерки по динамике стохастических систем, УРСС, М., 2012. 
[20] И. М. Лифшиц, С.А. Гредескул, Л.А. Пастур, Введение в теорию неупорядоченных систем, Наука, М., 1982.

[21] В. И. Кляцкин, А. И. Саичев, УФН, 162:3 (1992), 161-194.

[22] А. С. Михайлов, И. В. Упоров, УФН, 144:9 (1984), 79-112.

[23] В. И. Кляцкин, А. И. Саичев, ЖЭТФ, 111:4 (1997), 1297-1313.

[24] В. И. Кляцкин, О. Г. Чхетиани, ЖЭТФ, 136:2 (2009), 400-412.

[25] В. И. Кляцкин, ТМФ, 172:3 (2012), 415-436.

[26] Л. Д. Ландау, Е. М. Лифшиц, Теоретическая физика, т. 8: Электродинамика сплошных сред, Наука, М., 1982.

[27] В. И. Кляцкин, УФН, 174:2 (2004), 177-195.

[28] В. И. Кляцкин, УФН, 178:4 (2008), 419-431.

[29] В. И. Кляцкин, УФН, 179:5 (2009), 547-553.

[30] В. И. Кляцкин, УФН, 182:11 (2012), 1235-1237.

[31] V.I. Klyatskin, "On the criterion of stochastic structure formation in random media", Chaos and Complex Systems, Proceedings of the 4th International Interdisciplinary Chaos Symposium (Antalya, Turkey, April 29 - May 2, 2012), eds. S. G. Stavrinides, S. Banerjee, S. H. Caglar, M. Ozer, Springer, Berlin, 2013, 69-74.

[32] В. У. Заворотный, В. И. Кляцкин, В. И. Татарский, ЖЭТФ, 73:2 (1977), 481-497. 